\title{
Apixaban Use in Patients with Protein $C$ and S Deficiency: A Case Series and Review of Literature
}

\author{
Khalid Al Sulaiman $\mathbb{D}^{1-4}$, Faisal Alsuwayyid ${ }^{2}$, Abdulrahman Alrashidi ${ }^{2}$, Mohammed Alhijris ${ }^{2}$, Faisal Almutairi ${ }^{2}$, \\ Fahad Alharthi ${ }^{2}$, Laila Carolina Abu Esba ${ }^{1-3}$, Ohoud Aljuhani ${ }^{4,5}$, Hisham A Badreldin ${ }^{1-3}$ \\ 'Pharmaceutical Care Department, King Abdulaziz Medical City, Riyadh, Saudi Arabia; ${ }^{2}$ College of Pharmacy, King Saud Bin Abdulaziz University for \\ Health Sciences, Riyadh, Saudi Arabia; ${ }^{3}$ King Abdullah International Medical Research Center, Biostatistics and Bioinformatics Department, Riyadh, \\ Saudi Arabia; ${ }^{4}$ Saudi Critical Care Pharmacy Research (SCAPE) Platform, Riyadh/Jeddah, Saudi Arabia; ${ }^{5}$ Department of Pharmacy Practice, Faculty of \\ Pharmacy, King Abdulaziz University, Jeddah, Saudi Arabia \\ Correspondence: Khalid Al Sulaiman, King Abdulaziz Medical City (KAMC) - Ministry of National Guard Health Affairs (MNGHA), Riyadh, Saudi \\ Arabia, Email alsulaimankh@hotmail.com
}

\begin{abstract}
Background: Historically, warfarin was the mainstay anticoagulant agent to manage patients presenting with thrombotic disorders caused by Protein C or S deficiency. Several direct oral anticoagulants (DOACs) were introduced over the past decade. They showed superiority over warfarin in patients with venous thromboembolism in many landmark trials. Insufficient data are available that examine the outcome of utilizing apixaban in patients with protein $\mathrm{S}$ deficiency induce thrombosis.

Case(s) Presentation: We reported the clinical outcomes of utilizing apixaban in four patients with systemic thrombosis caused by protein C or S deficiency who presented to a tertiary hospital in Riyadh, Saudi Arabia. Four patients exhibited typical features of thrombotic events. After confirming the diagnosis, one patient was initially started on apixaban, and the other three patients were converted from warfarin to apixaban. Three of the four patients tolerated the apixaban during the follow-up period. Additionally, they did not have any bleeding or thrombotic complications. However, one patient developed recurrent thrombotic events despite switching to different type of DOAC and was ultimately transitioned back to warfarin.

Conclusion: Based on the available emerging evidence and our case series, the use of apixaban could be effective in preventing recurrent thrombotic events in patients with inherited thrombophilia without safety concerns. Further, large studies are warranted to investigate the safety and efficacy of apixaban in these population.
\end{abstract}

Keywords: apixaban, protein $\mathrm{S}$ deficiency, thrombosis, bleeding

\section{Introduction}

Several endogenous proteins such as protein $\mathrm{S}$, protein $\mathrm{C}$, and antithrombin are known to maintain the hemostatic equilibrium. ${ }^{1}$ The deficiency of any of these proteins could disrupt the hemostatic equilibrium and eventually cause venous thromboembolism (VTE). ${ }^{1}$ These deficiencies might be acquired or inherited as autosomal dominant traits. ${ }^{1}$ There are other types of thrombophilia, such as Factor V Leiden mutation, homocystinuria, and prothrombin G20210A. ${ }^{2}$ Individuals with hereditary protein $\mathrm{C}$ or $\mathrm{S}$ deficiency are more prone to develop VTE by approximately 11-folds compared to individuals without protein $\mathrm{C}$ or $\mathrm{S}$ deficiency. ${ }^{3}$

VTE manifested as either deep vein thrombosis (DVT), pulmonary embolism (PE), or both. Patients with inherited thrombophilia may require receiving anticoagulation therapy as primary or secondary prevention. ${ }^{1,4}$ Historically, vitamin $\mathrm{K}$ antagonists (VKAs) such as warfarin were used to treat inherited thrombophilia's induced VTE. ${ }^{5}$ Over the past decades, several large randomized clinical trials showed that most of direct oral anticoagulants (DOACs) are as effective as warfarin with better safety profile. ${ }^{6-8}$ These drugs were developed to address some of the primary drawbacks of warfarin, such as inter-individual variability, the prolonged time to attain full therapeutic effect, the narrow therapeutic index, the frequent coagulation monitoring, and the complex medication and food interactions. ${ }^{9}$ Moreover, several 
studies showed that DOACs may possess an incremental cost-effectiveness ratio and better quality of life for the treatment and prevention of VTE compared to warfarin therapy. ${ }^{10,11}$

Most of the studies that evaluated the efficacy and safety of DOACs in this subset of the patient population were small and predominantly included patients with low risk for thrombophilia (Factor V Leiden and prothrombin G20210A). Furthermore, the use of apixaban in hereditary thrombophilia, specifically high-risk thrombophilia (protein S deficiency), is controversial due to the lack of solid evidence. This case series aims to represent the experience of using apixaban in four patients with protein $\mathrm{C}$ and $\mathrm{S}$ deficiency.

\section{Case \#I}

In our first patient case, we describe a 41-year-old woman known to have chronic kidney disease (CKD) and peptic ulcer disease (PUD). Protein S deficiency was detected upon presentation with an extensive portal vein and superior mesenteric vein thrombosis and prior to initiating this patient on any anticoagulant. As a result, warfarin was initiated, targeting an International Normalized Ratio (INR) range of 2-3. She continued on warfarin for nine months. Her blood test showed a decreased protein S activity (22.8\%). During the duration of treatment with warfarin, she presented to the emergency department four times. She was treated for bleeding and supratherapeutic INR levels. A multidisciplinary decision was made to switch her from warfarin to apixaban $2.5 \mathrm{mg}$ BID. She remained on apixaban for approximately three years with no recurrent thrombotic or bleeding events up until her most recent follow-up (Tables 1 and 2).

\section{Case \#2}

In our second case, we describe a 49-year-old man with a body mass index of $33.5 \mathrm{~kg} / \mathrm{m}^{2}$ who was medically free. However, he presented to the emergency department with shortness of breath, pain in the lower extremity, and coughing blood-streaked sputum. He was diagnosed with an unprovoked DVT and PE. His protein S activity was $44.5 \%$, and he was initiated on apixaban with a loading dose of $10 \mathrm{mg}$ BID for seven days and continued on a maintenance dose of $5 \mathrm{mg}$ BID. He had no thrombotic or bleeding events for over five years and up until the date of this paper (Tables 1 and 2).

\section{Case \#3}

In our third case, we describe a 36-year-old man known to have protein S deficiency detected on presentation with a DVT at a young age (17 years). He had extremely decreased protein S activity (20\%). He was treated with warfarin for over 14 years until another DVT occurred and was switched to rivaroxaban $20 \mathrm{mg}$ once daily. He remained on rivaroxaban for approximately four years until he developed a PE. Then he was switched to apixaban $2.5 \mathrm{mg}$ BID, later his dose was increased to $5 \mathrm{mg}$ BID. Two years later, he developed a massive PE requiring intensive care unit admission, and he was switched back to warfarin at that time. Despite the patient adherence, no reason was identified for his recurrent VTE events while being on apixaban therapy. (Tables 1 and 2).

\section{Case \#4}

Our last case is a 51-year-old woman known to have a low protein C activity (49\%) and protein S activity (25\%) with a prior history of cerebral venous thrombosis on lifelong anticoagulation using warfarin. She was on warfarin before the cerebral thrombosis event and remained on it after the event. However, a decision was made to switch her to apixaban $5 \mathrm{mg}$ BID after developing subarachnoid hemorrhage and having reports of supratherapeutic and fluctuating INR levels with warfarin. For approximatly five years period and until her most recent follow-up she had no thrombotic or bleeding events while on apixaban (Tables 1 and 2).

\section{Discussion}

In this case series, we presented the data for four patients with protein $\mathrm{C}$ or S deficiency complicated by VTE or cerebral venous thrombosis maintained on apixaban. Three of the presented cases remained free from thrombotic or bleeding events for several years after apixaban initiation. However, one of the cases developed a new thrombotic event that required an alternative anticoagulant. 
Table I Baseline Characteristics

\begin{tabular}{|c|c|c|c|c|}
\hline Demographic Data & Case \#I & Case \#2 & Case \#3 & Case \#4 \\
\hline Age (years) & 41 & 49 & 36 & 51 \\
\hline BMI $\left(\mathrm{kg} / \mathrm{m}^{2}\right)$ & 32.47 & 33.51 & 32.4 & 34.13 \\
\hline Gender & Female & Male & Male & Female \\
\hline Weight (kg) & 77 & 98 & 112 & 82 \\
\hline Blood group & $\mathrm{O}+$ & NA & B- & NA \\
\hline \multicolumn{5}{|l|}{ Co-existing illness } \\
\hline Hypertension (HTN) & No & No & Yes & Yes \\
\hline Asthma & No & No & No & Yes \\
\hline $\begin{array}{l}\text { Chronic obstructive pulmonary disease } \\
\text { (COPD) }\end{array}$ & No & No & No & No \\
\hline Diabetes mellitus (DM) & No & No & No & Yes \\
\hline Chronic kidney disease (CKD) & Yes & No & No & No \\
\hline Ischemic heart disease (IHD) & No & No & No & No \\
\hline Atrial fibrillation (AFib or AF) & No & No & No & No \\
\hline Heart failure (HF) & No & No & No & No \\
\hline Acute coronary syndrome (ACS) & No & No & No & No \\
\hline Dyslipidemia (DLP) & No & No & No & Yes \\
\hline CVA (Stroke) & No & No & No & Yes \\
\hline Hypothyroidism & No & No & No & No \\
\hline Valvular disease & No & No & No & No \\
\hline Venous thromboembolism (VTE) & No & Yes & Yes (PE) & No \\
\hline CABG & No & No & No & No \\
\hline Liver disease (any) & No & No & No & No \\
\hline Peptic ulcer disease & Yes & No & No & No \\
\hline Systemic lupus erythematosus (SLE) & No & No & No & No \\
\hline $\begin{array}{l}\text { Antiphospholipid antibody syndrome } \\
\text { (APLS) }\end{array}$ & No & No & No & No \\
\hline Cancer & No & No & No & No \\
\hline History of major bleeding & No & No & No & No \\
\hline \multicolumn{5}{|l|}{ Baseline Labs } \\
\hline Protein $\mathrm{S}$ activity & $22.8 \%$ & $45.5 \%$ & $20 \%$ & $25.1 \%$ \\
\hline Protein $\mathrm{C}$ activity & NA & NA & NA & $49 \%$ \\
\hline Serum creatinine $(\mu \mathrm{mol} / \mathrm{l})$ & 90 & 88 & 90 & 55 \\
\hline Blood urea nitrogen $(\mathrm{BUN})(\mathrm{mmol} / \mathrm{l})$ & 6.7 & 4.9 & NA & 3 \\
\hline
\end{tabular}


Table I (Continued).

\begin{tabular}{|c|c|c|c|c|}
\hline Demographic Data & Case \# I & Case \#2 & Case \#3 & Case \#4 \\
\hline Total bilirubin $(\mu \mathrm{mol} / \mathrm{L})$ & 5 & NA & 10.2 & 5.7 \\
\hline INR & 1.97 & 1.03 & 1.75 & 5.09 \\
\hline Platelets count $\left(10^{9} / \mathrm{L}\right)$ & 317 & 239 & 259 & 415 \\
\hline $\begin{array}{l}\text { Activated partial thromboplastin time } \\
\text { (aPTT) (seconds) }\end{array}$ & NA & NA & 37.1 & 57.4 \\
\hline Alanine aminotransferase (ALT) (U/L) & 13 & 29 & 41 & 43 \\
\hline Aspartate aminotransferase (AST) (U/L) & 17 & 24 & 108 & 24 \\
\hline Albumin (gm/L) & 31 & $4 I$ & 44 & 40 \\
\hline White blood cells (WBCs) $\left(10^{9} / \mathrm{L}\right)$ & 7.44 & 6.4 & 4.85 & 14.16 \\
\hline Blood glucose level (mmol/l) & 5.9 & 4.8 & NA & 8 \\
\hline Hematocrit (Hct) (L/L) & 0.33 & 0.45 & 0.47 & 0.37 \\
\hline Fibrinogen $(\mathrm{gm} / \mathrm{L})$ & 3.17 & NA & NA & NA \\
\hline D-dimer (mg/l) & 1.79 & 6.47 & NA & NA \\
\hline Apixaban use & Yes & Yes & Yes & Yes \\
\hline Date of initiation & $28 / 06 / 2018$ & $24 / 11 / 2016$ & 2018 & $05 / 10 / 2016$ \\
\hline Dose of apixaban & $5 \mathrm{mg}$ & $10 \mathrm{mg}$ & $\begin{array}{l}5 \mathrm{mg} \text { (initially) then } \\
\text { increased to } 10 \mathrm{mg}\end{array}$ & $10 \mathrm{mg}$ \\
\hline $\begin{array}{l}\text { Concomitant antiplatelets use (ie, aspirin, } \\
\text { clopidogrel, ticagrelor) }\end{array}$ & No & No & No & No \\
\hline Gl prophylaxis & Yes (PPI) & Yes (PPI) & No & Yes \\
\hline
\end{tabular}

Table 2 Outcomes after Apixaban initiation

\begin{tabular}{|c|c|c|c|c|}
\hline Outcomes & Case \# I & Case \#2 & Case \#3 & Case \#4 \\
\hline Major bleeding & No & No & No & No \\
\hline Minor bleeding & No & No & No & No \\
\hline Hospital admission within 6 months of apixaban initiation & Yes & Yes & No & Yes \\
\hline Hospital admission within 12 months of apixaban initiation & Yes & Yes & No & Yes \\
\hline New stroke within 6 months of apixaban initiation & No & No & No & No \\
\hline Any new stroke after apixaban initiation & No & No & No & No \\
\hline New VTE within 6 months of Apixaban initiation & No & No & No & No \\
\hline Any new VTE after Apixaban initiation & No & No & Yes (PE) & No \\
\hline Any new thrombosis after apixaban initiation & No & No & Yes (PE) & No \\
\hline Switched to other anticoagulants (eg, warfarin) & No & No & Yes (Warfarin) & No \\
\hline
\end{tabular}


Published guidelines did not make a firm statement regarding the anticoagulation modality and duration in patients with thrombophilia due to limited evidence. The American Society of Hematology (ASH) has provided a conditional recommendation for a shorter course (3-6 months) for the primary treatment of unprovoked VTE in patients with more than one nonenvironmental risk factor for recurrent VTE. These risk factors included inherited thrombophilia, older age, and male gender. Following the primary treatment course, the ASH suggested indefinite antithrombotic therapy over stopping anticoagulation. Moreover, they recommended an annual visit to review the clinical indication for indefinite antithrombotic therapy as well as any complications for the secondary prevention of recurrent VTE. ${ }^{4}$

Secondary prevention of VTE with VKA could be challenging due to frequent laboratory testing, narrow therapeutic index, complex dosage regimens, and drug-drug or drug-food interactions. Apixaban has several advantages over warfarin as having similar efficacy profile with minimal risk of bleeding ${ }^{12}$ It could be an appealing option for patients with protein $\mathrm{C}$ or $\mathrm{S}$ deficiency requiring prolonged anticoagulation therapy. Protein $\mathrm{S}$ deficiency patients are known to have complex anticoagulation management that further complicate the appropriate choice of anticoagulants agent. Several reports evaluated the use of DOACs in patients with thrombophilia and showed relatively positive efficacy and safety outcomes. However, the majority of the available evidence in this field are originating from case studies and retrospective cohorts with numerous confounding factors.

Skelley et al conducted a systematic review to evaluate the use of DOACs in patients with hypercoagulable states related to thrombophilia. ${ }^{13}$ Six case studies were included in which dabigatran was used in one case, and rivaroxaban was used in the remaining five cases. Overall, all patients did not develop recurrent VTE episodes except one patient who developed a thrombosis in the popliteal and tibial veins. The only clinical trial included in that review was a post-hoc, sub-group analysis conducted on the data from the RE-MEDY trial. The authors concluded that the use of dabigatran in patients with thrombophilia demonstrated non-inferiority compared to warfarin in terms of recurrent VTE or VTE-related death. ${ }^{13}$ Consistent with our case series, a follow-up study conducted on 45 patients with severe inherited thrombophilia, found that DOACs were safe and effective for treating 18 patients with protein S deficiency with VTE. ${ }^{14}$ In these 45 patients, both rivaroxaban and apixaban showed no thromboembolism, stroke or major bleeding during the 29-month follow-up period. In agreement with this study, we reported no stroke events for at least six months of apixaban initiation in the four cases.

Additionally, none of our cases developed minor or major bleeding after apixaban use. In line with our report, a recent meta-analysis showed no difference in major bleeding between patients treated with DOACs compared to VKAs, including those with protein $\mathrm{S}$ deficiencies. ${ }^{15}$ This meta-analysis reviewed studies involving rivaroxaban, dabigatran, and edoxaban but did not include any study related to apixaban use in protein $\mathrm{S}$ deficiency.

On the other hand, a single-center cohort study was conducted on 56 VTE patients that included 12 patients with protein S deficiency in which apixaban was used in $25 \%$ of them. ${ }^{16}$ This study reported one non-fatal VTE that was observed in one patient on apixaban. Additionally, this study showed that major bleeding and clinically relevant nonmajor bleeding occurred in only six patients $(10.7 \%)$, in which two of them were on apixaban. ${ }^{16}$ Similar to our conclusion, this study concluded that full-dose apixaban is effective and safe in patients with severe inherited thrombophilia including protein $\mathrm{S}$ deficiency. However, the degree of protein $\mathrm{S}$ deficiency needs to be assessed prior to apixaban use in order to accurately link it with the clinical outcomes. Further, large studies are warranted to explicitly investigate the safety and efficacy of apixaban in this subset of patients. Our case series is the first to describe the use of these agents in a Saudi individuals. Based on our repeorted cases, the use of apixaban as alternative to warfarin is feasible and might be safe and effective.

\section{Conclusion}

Based on the available emerging evidence and our case series, the use of apixaban could be effective in preventing recurrent thrombotic events in patients with inherited thrombophilia without safety concerns. Larger randomized clinical trials are warranted to confirm our finding. 


\section{Data Sharing Statement}

The datasets used and/or analyzed during the current study are available from corresponding author on reasonable request.

\section{Ethics Approval and Consent to Participate}

The study was approved by King Abdullah International Medical Research Center Institutional Review Board, Riyadh, Saudi Arabia (Reference No: NRC21R/550/12). Participants' confidentiality was strictly observed throughout the study by using anonymous unique serial numbers for each subject and restricting data only to the investigators. Written informed consent was obtained from the four patients for publication of this case series and any accompanying images. A copy of the written consent is available for review by the Editor-in-Chief of this journal.

\section{Acknowledgments}

We would like to acknowledge the investigators in the Saudi Critical Care Pharmacy Research (SCAPE) platform who participated in this project.

\section{Author Contributions}

All authors made a significant contribution to the work reported, whether that is in the conception, study design, execution, acquisition of data, analysis and interpretation, or in all these areas; took part in drafting, revising or critically reviewing the article; gave final approval of the version to be published; have agreed on the journal to which the article has been submitted; and agree to be accountable for all aspects of the work.

\section{Disclosure}

The authors declare that they have no competing interests in this work.

\section{References}

1. Rosendaal FR. Venous thrombosis: a multicausal disease. Lancet. 1999;353(9159):1167-1173. doi:10.1016/s0140-6736(98)10266-0

2. Khan S, Dickerman JD. Hereditary thrombophilia. Thromb J. 2006;4(1):15. doi:10.1186/1477-9560-4-15

3. De Stefano V, Finazzi G, Mannucci PM. Inherited thrombophilia: pathogenesis, clinical syndromes, and management. Blood. 1996;87 (9):3531-3544. doi:10.1182/blood.V87.9.3531.bloodjournal8793531

4. Ortel TL, Neumann I, Ageno W, et al. American Society of Hematology 2020 guidelines for management of venous thromboembolism: treatment of deep vein thrombosis and pulmonary embolism. Blood Adv. 2020;4(19):4693-4738. doi:10.1182/bloodadvances.2020001830

5. Lipe B, Ornstein DL. Deficiencies of natural anticoagulants, protein C, protein S, and antithrombin. Circulation. 2011;124(14):e365-e368. doi:10.1161/CIRCULATIONAHA.111.044412

6. Agnelli G, Becattini C, Meyer G, et al. Apixaban for the treatment of venous thromboembolism associated with cancer. $N$ Engl J Med. 2020;382 (17):1599-1607. doi:10.1056/NEJMoa1915103

7. Büller HR, Décousus H, Grosso MA, et al. Edoxaban versus warfarin for the treatment of symptomatic venous thromboembolism. $N$ Engl $J$ Med. 2013;369(15):1406-1415. doi:10.1056/NEJMoa1306638

8. Schulman S, Kearon C, Kakkar AK, et al. Dabigatran versus Warfarin in the treatment of acute venous thromboembolism. N Engl J Med. 2009;361 (24):2342-2352. doi:10.1056/NEJMoa0906598

9. Pirmohamed M. Warfarin: almost 60 years old and still causing problems. Br J Clin Pharmacol. 2006;62(5):509-511. doi:10.1111/j.13652125.2006.02806.x

10. Al Mukdad M, Al-Badriyeh D, Elewa HF. Cost-effectiveness evaluations among the direct oral anticoagulants for the prevention and treatment of venous thromboembolism: systematic review. Clin Appl Thromb. 2019;25:1076029619849103. doi:10.1177/1076029619849103

11. Keita I, Aubin-Auger I, Lalanne C, et al. Assessment of quality of life, satisfaction with anticoagulation therapy, and adherence to treatment in patients receiving long-course vitamin $\mathrm{K}$ antagonists or direct oral anticoagulants for venous thromboembolism. Patient Prefer Adherence. 2017;11:1625-1634. doi:10.2147/PPA.S131157

12. Burn J, Pirmohamed M. Direct oral anticoagulants versus warfarin: is new always better than the old? Open Hear. 2018;5(1):e000712. doi:10.1136/ openhrt-2017-000712

13. Skelley JW, White CW, Thomason AR. The use of direct oral anticoagulants in inherited thrombophilia. J Thromb Thrombolysis. 2017;43 (1):24-30. doi:10.1007/s11239-016-1428-2

14. Serrao A, Lucani B, Mansour D, et al. Direct oral anticoagulants in patients affected by major congenital thrombophilia. Mediterr J Hematol Infect Dis. 2019;11(1):e2019044. doi:10.4084/MJHID.2019.044

15. Elsebaie MAT, van Es N, Langston A, Büller HR, Gaddh M. Direct oral anticoagulants in patients with venous thromboembolism and thrombophilia: a systematic review and meta-analysis. J Thromb Haemost. 2019;17(4):645-656. doi:10.1111/jth.14398

16. Zuk J, Papuga-Szela E, Zareba L, Undas A. Direct oral anticoagulants in patients with severe inherited thrombophilia: a single-center cohort study. Int J Hematol. 2021;113(2):190-198. doi:10.1007/s12185-020-03012-7 


\section{Publish your work in this journal}

The Journal of Blood Medicine is an international, peer-reviewed, open access, online journal publishing laboratory, experimental and clinical aspects of all aspect pertaining to blood based medicine including but not limited to: Transfusion Medicine; Blood collection, Donor issues, Transmittable diseases, and Blood banking logistics; Immunohematology; Artificial and alternative blood based therapeutics; Hematology; Biotechnology/nanotechnology of blood related medicine; Legal aspects of blood medicine; Historical perspectives. The manuscript management system is completely online and includes a very quick and fair peer-review system. Visit http://www.dovepress.com/testimonials.php to read real quotes from published authors.

Submit your manuscript here: http://www.dovepress.com/journal-of-blood-medicine-journal 